\title{
Nonthermal Electrons in Radiatively Inefficient Accretion Flow Models of Sagittarius A*
}

\section{Citation}

Yuan, Feng, Eliot Quataert, and Ramesh Narayan. 2003. "Nonthermal Electrons in Radiatively Inefficient Accretion Flow Models of Sagittarius A*." The Astrophysical Journal 598 (1): 301-12. https://doi.org/10.1086/378716.

\section{Permanent link}

http://nrs.harvard.edu/urn-3:HUL.InstRepos:41384950

\section{Terms of Use}

This article was downloaded from Harvard University's DASH repository, and is made available under the terms and conditions applicable to Other Posted Material, as set forth at http:// nrs.harvard.edu/urn-3:HUL.InstRepos:dash.current.terms-of-use\#LAA

\section{Share Your Story}

The Harvard community has made this article openly available.

Please share how this access benefits you. Submit a story. 


\title{
Nonthermal Electrons in Radiatively Inefficient Accretion Flow Models of Sagittarius A*
}

\author{
Feng Yuan \\ Harvard-Smithsonian Center for Astrophysics, 60 Garden Street, Cambridge, MA 02138 \\ fyuan@cfa.harvard.edu \\ Eliot Quataert \\ Astronomy Department, 601 Campbell Hall, University of California, Berkeley, CA 94720 \\ eliot@astron. berkeley.edu \\ Ramesh Narayan \\ Harvard-Smithsonian Center for Astrophysics, 60 Garden Street, Cambridge, MA 02138 \\ narayan@cfa.harvard.edu
}

\begin{abstract}
We investigate radiatively inefficient accretion flow models for Sgr A*, the supermassive black hole in our Galactic Center, in light of new observational constraints. Confirmation of linear polarization in the submm emission argues for accretion rates much less than the canonical Bondi rate. We consider models with low accretion rates and calculate the spectra produced by a hybrid electron population, consisting of both thermal and nonthermal particles. The thermal electrons produce the submm emission and can account for its linear polarization properties. As noted in previous work, the observed low-frequency radio spectrum can be explained if a small fraction $(\approx 1.5 \%)$ of the electron thermal energy resides in a soft power-law tail. In the innermost region of the accretion flow, turbulence and/or magnetic reconnection events may occasionally accelerate a fraction of the electrons into a harder power-law tail. We show that the synchrotron emission from these electrons, or the Compton up-scattering of synchrotron photons by the same electrons, may account for the X-ray flares observed by Chandra.
\end{abstract}

Subject headings: accretion, accretion disks — black hole physics — galaxies: active - Galaxy: center — radiation mechanisms: thermal — radiation mechanisms: non-thermal 


\section{Introduction}

There is compelling evidence that the center of our Galaxy hosts a massive black hole (BH) with mass $M=2.6 \times 10^{6} M_{\odot}$ (e.g., Schödel et al. 2002; Ghez et al. 2003). The inferred location of the black hole is coincident with the energetic radio source Sgr A* (e.g., Melia \& Falcke 2001), which has been intensively studied since its discovery. The radio spectrum of Sgr A* consists of two components which dominate below and above $86 \mathrm{GHz}$, respectively. The component below $86 \mathrm{GHz}$ has a spectrum $F_{\nu} \propto \nu^{0.2}$, while the high frequency component, the "submm bump", has a spectrum $F_{\nu} \propto \nu^{0.8}$ up to $\sim 10^{3} \mathrm{GHz}$ (Zylka, Mezger \& Lesch 1992; Serabyn et al. 1997; Falcke et al. 1998; Zhao et al. 2003). At yet higher frequencies, in the IR, there are strong upper limits to the flux, indicating that the spectrum cuts-off steeply (e.g., Hornstein et al. 2002). The IR limit plus an X-ray detection from Chandra (Baganoff et al. 2001, 2003) indicate that $\mathrm{Sgr} \mathrm{A}$ is quite dim overall, with a bolometric luminosity of only $L \approx 10^{36} \mathrm{ergs} \mathrm{s}^{-1} \approx 3 \times 10^{-9} L_{\mathrm{Edd}}$. Most of this luminosity is radiated in the submm bump.

The proximity of the Galactic Center allows one to determine observationally the dynamics of gas quite close to the $\mathrm{BH}$, providing unique constraints on theoretical models of the accretion flow. A canonical estimate for the rate at which a $\mathrm{BH}$ gravitationally captures surrounding gas is given by the spherical accretion model of Bondi (1952). This model can be applied directly to Sgr A* (see Melia 1992 for an early application and Melia, Liu, \& Coker 2001 for a current version of the spherical accretion model). Chandra observations of the Galactic Center detect extended diffuse emission within $1-10^{\prime \prime}$ of the BH (Baganoff et al. 2003). This emission likely arises from hot gas produced when the winds from massive stars collide and shock. Interpreted as such, the inferred gas density and temperature are $\approx 130 \mathrm{~cm}^{-3}$ and $\approx 2 \mathrm{keV}$ about $1^{\prime \prime}$ from the BH. A remarkable coincidence is that, given the measured $\mathrm{BH}$ mass and measured ambient temperature, the sphere of influence of the $\mathrm{BH}$ (defined by the Bondi accretion radius) is $R_{a c c} \approx G M / c_{\infty}^{2} \approx 0.04 \mathrm{pc} \approx 1^{\prime \prime}$, comparable to Chandra's resolution! The Bondi accretion rate is thus very well determined: $\dot{M}_{B} \approx 10^{-5} M_{\odot} \mathrm{yr}^{-1}$. If gas were accreting at this rate onto the $\mathrm{BH}$ via a thin accretion disk (Shakura \& Sunyaev 1973), a model that has been extensively and successfully applied to luminous accreting sources (e.g., Koratkar \& Blaes 1999), the expected luminosity would be $L \approx 0.1 \dot{M}_{B} c^{2} \approx 10^{41} \mathrm{ergs} \mathrm{s}^{-1}$. This is larger than the observed bolometric luminosity of Sgr $\mathrm{A}^{*}$ by a factor of $\sim 10^{5}$. The observations thus favor accretion models in which very little

of the gravitational potential energy of the inflowing gas is radiated, i.e., $L \ll \dot{M}_{B} c^{2}$. We will refer to such models as radiatively inefficient accretion flows (RIAFs). The distinction between a Bondi accretion flow and a RIAF is that the former describes a spherical flow with no angular momentum while the latter describes a hot quasi-spherical rotating accretion flow with viscosity. 
Advection-dominated accretion flows (ADAFs) are an analytically-motivated model for the dynamics of RIAFs (e.g., Narayan \& Yi 1994). In such models, $\dot{M} \approx \alpha \dot{M}_{B}$ (where $\alpha \sim 0.1$ is the dimensionless viscosity parameter) and $\rho \propto r^{-3 / 2}$, as in spherical Bondi accretion. A number of studies have shown that ADAF models accreting at the observationally inferred rate could roughly account for the spectrum and luminosity of Sgr A* (Narayan et al. 1995, 1998; Manmoto et al. 1997; Mahadevan 1998; Özel, Psaltis \& Narayan 2000; Narayan 2002). The key requirement in these models is that the fraction of the turbulent energy heating the electrons, $\equiv \delta$, has to be quite small, $\delta \lesssim 0.01$, in order to explain the low luminosity of Sgr $\mathrm{A}^{*}$; the corresponding electron temperature close to the $\mathrm{BH}$ is $T_{e} \sim 10^{10} \mathrm{~K} \ll T_{p} \sim 10^{12} \mathrm{~K}$.

In this paper we reexamine RIAF models for the spectrum of Sgr A*. We are motivated both by important new observational results on the emission from Sgr A* and by theoretical work which shows that the analytical ADAF model does not accurately describe the dynamics of RIAFs. We first summarize the new observational constraints.

Recent radio observations show that Sgr A* has no linear polarization between 1.4 and $112 \mathrm{GHz}$ with limits of 0.1 to $2 \%$ (Bower et al. 1999a,b, 2001), while the source has measurable circular polarization between 1.4 and $43 \mathrm{GHz}$ (Bower, Falcke, \& Backer 1999). At higher frequencies, JCMT observations at 150, 220, 375 and $400 \mathrm{GHz}$ indicate that Sgr A* is linearly polarized at a level of $\sim 10 \%$ (Aitken et al. 2000). This result was confirmed by the BIMA array, which has a much better angular resolution - Bower et al. (2003) detected a linear polarization of $7.2 \pm 0.6 \%$ at $230 \mathrm{GHz}$. This observation alone places an upper limit on the rotation measure of $2 \times 10^{6} \mathrm{rad} \mathrm{m}^{-2}$. In an ADAF model, as originally formulated, the region where the (linearly polarized) submm-bump is predicted to originate has such a large electron density that the rotation measure would be many orders of magnitude larger than the above observational upper limit (Quataert \& Gruzinov 2000; Agol 2000). Assuming that the magnetic field does not undergo many reversals along the line of sight (see Ruszkowski \& Begelman 2002), the polarization data thus impose a serious constraint on models of the accretion flow.

At X-ray wavelengths, Chandra has convincingly detected an X-ray source coincident with Sgr $\mathrm{A}^{*}$ (to within $\approx 0.2^{\prime \prime}$, which is $\approx 0.01$ pc or $\approx 3 \times 10^{4} R_{S}$ at the Galactic Center, where $R_{S}$ is the Schwarzschild radius of the $\left.\mathrm{BH}\right)$. Chandra observations show that this $\mathrm{X}$ ray source comes in two states. In the quiescent state, the absorption-corrected $2-10 \mathrm{keV}$ luminosity is $2.2_{-0.3}^{+0.4} \times 10^{33} \mathrm{erg} \mathrm{s}^{-1}$. The spectrum is soft and is well fitted by an absorbed power-law model with photon index $\Gamma=2.2_{-0.7}^{+0.5}$. A large fraction of the X-ray flux comes from an extended region with diameter $\approx 1.4^{\prime \prime}$. Comparisons between two observations separated by about a year show that this component remains constant (Baganoff et al. 2003). In the flare state, the luminosity of Sgr A* increases by a factor of few to 45 over a timescale of 
minutes to hours; the short timescale argues that the emission arises quite close to the $\mathrm{BH}$, roughly within $\lesssim 10-100 R_{S}$. For the strongest flare, the luminosity is $\approx 10^{35} \mathrm{erg} \mathrm{s}^{-1}$, the timescale is about 3 hours, and the spectrum is hard, $\Gamma=1.3_{-0.6}^{+0.5}$ (Baganoff et al. 2001). Further observations by both XMM-Newton (Goldwurm et al. 2003) and Chandra (Baganoff 2003a) indicate that rapid and intense X-ray flares, with a factor of five or more increase in luminosity, are relatively common and occur roughly once per day. Such flares provide important constraints on the gas dynamics close to the $\mathrm{BH}$.

To search for counterparts to the X-ray flares, a multiwavelength campaign was conducted, including radio, submm, IR, and X-ray observations (Baganoff 2003a). The X-ray flares are apparently not accompanied by large variations at longer wavelengths, though there may be some evidence for variations at $\mathrm{mm}$ wavelengths at the level of a few tens of percent.

In addition to these new observational constraints, there has been a significant change in the theoretical understanding of RIAFs over the past few years. Most importantly, global, time-dependent, numerical simulations reveal that the structure of the flow is very different from the original self-similar ADAF prediction of Narayan \& Yi (1994). The key result from nearly all such simulations is that $\dot{M} \ll \dot{M}_{B}$, i.e., very little mass available at large radii actually accretes onto the black hole; most of it is lost to a magnetically driven outflow or circulates in convective motions (e.g., Stone, Pringle, \& Begelman 1999; Igumenshchev \& Abramowicz 1999; Igumenshchev et al. 2000; Stone \& Pringle 2001; Hawley \& Balbus 2002; Igumenshchev et al. 2003). Another way to state this result is that for a given gas density at a large distance from the black hole (e.g., measured by Chandra on 1" scales), the density close to the $\mathrm{BH}$ is much less than the ADAF or Bondi predictions. This theoretical result is consistent with the linear polarization detection from Sgr A*, which argues strongly for low gas densities close to the $\mathrm{BH}$ and thus low $\dot{M} \ll \dot{M}_{B}$.

Quataert \& Narayan (1999) showed that RIAF models with $\dot{M} \ll \dot{M}_{B}$ could account for the basic observed properties of Sgr $A^{*}$. To produce the correct amount of emission, however, their models required that the electrons in the accretion flow should be hotter than in ADAF models ( $\sim 10^{11} \mathrm{~K}$ close to the $\mathrm{BH}$ rather than $10^{10} \mathrm{~K}$ ). Stated another way, $\delta$, the fraction of the turbulent energy that heats the electrons, must be larger. Given the theoretical uncertainties in how electrons are heated in the accretion flow, this is very plausible (e.g., Quataert \& Gruzinov 1999). However, in the work of Quataert \& Narayan (1999), old X-ray spectral data were used and the accretion rate at the outer boundary was treated as a free parameter. Given the considerable progress in the field during the last few years, it is neccesary to check whether a RIAF model can fit the new data, specifically, (i) the Chandra X-ray spectrum, (ii) the measured gas density at the accretion radius (from 
Chandra), and (iii) the gas density near the $\mathrm{BH}$ (from radio polarization data). This is the goal of the present paper.

An additional issue is the electron distribution function. Since the inflowing gas is collisionless, processes such as MHD turbulence, reconnection, and weak shocks can accelerate electrons and generate a nonthermal tail at high energies in the electron distribution function. The effect of such nonthermal electrons on the synchrotron spectrum of Sgr A* was investigated by Mahadevan (1998) and Özel et al. (2000) (see Wardziński \& Zdziarski 2001 for a discussion of the effect of a power-law tail on synchrotron and inverse Compton emissions in luminous systems). They found that the low-frequency radio spectrum, which was under-predicted in earlier ADAF models (e.g., Narayan et al. 1998), could be explained quite well, if roughly $1 \%$ of the steady state electron energy is in nonthermal electrons ${ }^{1}$. However, the calculations were based on a model with a high accretion rate in the inner region of the accretion flow, $\dot{M} \approx 3 \times 10^{-6} M_{\odot} \mathrm{yr}^{-1}$, which is about two orders of magnitude larger than the upper limit on the accretion rate implied by the radio linear polarization measurements. Therefore, a secondary goal of the present paper is to examine how the predictions for the radio emission are modified when the density in the inner region of the accretion flow is much lower than previously considered.

In the calculations reported here we focus in particular on non-thermal electrons, which can account for the low frequency radio emission and which may also be responsible for the X-ray flares seen by Chandra. In $\S 2$, we present our method for treating a nonthermal electron distribution function in the accretion flow. In $\S 3$, we present our results of fitting the quiescent spectrum of Sgr $A^{*}$. Then, in $\S 4$, we consider possible origins of the X-ray flares observed in Sgr A*. We conclude with a summary and discussion in $§ 5$.

\section{Methodology}

\subsection{Dynamics of the Accretion Flow}

To take into account the role of outflows/convection in modifying the density profile of the RIAF, we write the dependence of $\dot{M}$ on radius as follows (Blandford \& Begelman 1999),

\footnotetext{
${ }^{1}$ Liu \& Melia (2001) reached a similar conclusion: they showed that the combination of centimeter and $\mathrm{X}$-ray data preclude the possibility of producing the observed $1.36 \mathrm{GHz}$ radio flux via thermal synchrotron emission in a bounded accretion flow and that nonthermal electrons are needed
} 


$$
\dot{M}=-4 \pi R H \rho v=\dot{M}_{\text {out }}\left(\frac{R}{R_{\text {out }}}\right)^{s},
$$

where $\dot{M}_{\text {out }}$ is the mass accretion rate at the outer boundary of the flow $\left(\sim \alpha \dot{M}_{B}\right), R_{\text {out }} \approx$ $R_{a c c} \approx 10^{5} R_{s}$ is the "outer radius" of the flow (the Bondi accretion radius), and $H, \rho$, and $v$ are the scale height, mass density, and radial velocity, respectively; we set $H=c_{s} / \Omega_{\mathrm{K}}$ where $c_{s}$ is the sound speed and $\Omega_{\mathrm{K}}$ is the Keperian angular velocity. The parameter $s$ describes how the density profile and accretion rate are modified. The net accretion rate onto the $\mathrm{BH}$ is $\dot{M}_{\text {in }} \approx \dot{M}_{\text {out }}\left(R_{\text {in }} / R_{\text {out }}\right)^{s}$; in a self-similar flow this would correspond to a density profile $\rho(r) \propto r^{-3 / 2+s}$.

We assume that a large fraction $\delta$ of the turbulent energy directly heats the electrons. Assuming for now that the electrons are thermal, the electron energy equation is given by

$$
\rho v\left(\frac{d \varepsilon_{e}}{d r}-\frac{p_{e}}{\rho^{2}} \frac{d \rho}{d r}\right)=\delta q^{+}+q_{i e}-q^{-},
$$

where $\varepsilon_{e}$ is the internal energy of electrons per unit mass of the gas, $p_{e}$ is the pressure due to electrons, $q_{i e}$ is the Coulomb energy exchange rate between electrons and ions, $q^{-}$is the electron cooling rate, and $q^{+}$is the net turbulent heating rate. The energy equation for the ions is then given by

$$
\rho v\left(\frac{d \varepsilon_{i}}{d r}-\frac{p_{i}}{\rho^{2}} \frac{d \rho}{d r}\right)=(1-\delta) q^{+}-q_{i e}=-(1-\delta) \alpha p r \frac{d \Omega}{d r}-q_{i e} .
$$

The other two equations, namely the radial and azimuthal components of the momentum equation, are

$$
\begin{gathered}
v \frac{d v}{d r}=-\Omega_{\mathrm{k}}^{2} r+\Omega^{2} r-\frac{1}{\rho} \frac{d p}{d r}, \\
v\left(\Omega r^{2}-j\right)=\alpha r \frac{p}{\rho} .
\end{gathered}
$$

Here, $j$ is the specific angular momentum of the gas accreted into the BH, $\Omega$ denotes the angular velocity, and $p=p_{i}+p_{e}$ is the total gas pressure (electron plus ion). We adopt the Paczyński \& Wiita (1980) potential to mimic the geometry of a Schwarzschild black hole.

We solve the above set of equations, with appropriate boundary conditions (e.g., Yuan 1999; Yuan et al. 2000), to obtain the flow characteristics such as ion and electron temperatures, density, etc. We should note that the equations solved here are not fully consistent since they do not account for any energy or angular momentum that is transported by convection or is lost to an outflow. We expect that this will not significantly change our results since the primary effect of outflows/convection is to modify the density profile of the flow, which we have accounted for (small additional uncertainties in, e.g., the temperature, are absorbed into the large uncertainties in the heating parameter $\delta$ ). 


\subsection{Electron Distribution Function}

We consider a hybrid distribution of electrons at each radius in the accretion flow. The thermal distribution is

$$
n_{\mathrm{th}}(\gamma)=\frac{N_{\mathrm{th}} \gamma^{2} \beta \exp \left(-\gamma / \theta_{e}\right)}{\theta_{e} \mathrm{~K}_{2}\left(1 / \theta_{e}\right)},
$$

where $\gamma$ is the electron Lorentz factor, and $\theta_{e} \equiv k T_{e} / m_{e} c^{2}$ is the dimensionless electron temperature. The power-law distribution is described by

$$
\begin{array}{cc}
n_{\mathrm{pl}}(\gamma)=N_{\mathrm{pl}}(p-1) \gamma^{-p}, & \gamma_{\min } \leq \gamma \leq \gamma_{c}, \\
n_{\mathrm{pl}}(\gamma)=N_{\mathrm{pl}}(p-1) \gamma_{c} \gamma^{-p-1}, & \gamma_{\mathrm{c}} \leq \gamma \leq \gamma_{\max },
\end{array}
$$

where $\gamma_{\min }, \gamma_{\max }$ and $\gamma_{\mathrm{c}}$ are the minimum, maximum, and the "cooling break" Lorentz factors, respectively. From the global solution for the accretion flow structure, we obtain the strength of the magnetic field, $B$, at each radius, assuming that the magnetic energy density is a fixed fraction $\beta^{-1}$ of the thermal energy density (an equipartition prescription). The "cooling break" Lorentz factor $\gamma_{c}$ is then determined by the condition

$$
t_{\text {cool }} \equiv \frac{3}{4} \frac{8 \pi m_{e} c}{\sigma_{\mathrm{T}} \gamma_{c} \beta_{e}^{2} B^{2}}=t_{\text {accretion }} \equiv \frac{R}{|v|}
$$

Usually, the cooled electrons have an energy index $(p+1)$ as written in equation (7b) (Throughout the paper, $p$ denotes the spectral index of the injected electrons in the powerlaw energy distribution). However, if $p<1$, the "cooled" electron distribution function goes as $n(\gamma) \propto \gamma^{-2}$, not $n(\gamma) \propto \gamma^{-p-1}$.

We calculate the values of $\gamma_{\min }$ and $N_{\mathrm{pl}}$ as follows. We assume that the injected energy in nonthermal electrons is equal to a fraction $\eta$ of the energy in thermal electrons; as a fiducial model, we take $\eta$ to be independent of radius (but see $\S 4$ where we consider X-ray flares). The energy density of thermal electrons at temperature $\theta_{e} \equiv k T_{e} / m_{e} c^{2}$ is (Chandrasekhar 1939)

$$
u_{\mathrm{th}}=a\left(\theta_{e}\right) N_{\mathrm{th}} m_{e} c^{2} \theta_{e}
$$

where the quantity

$$
a\left(\theta_{e}\right) \equiv \frac{1}{\theta_{e}}\left[\frac{3 K_{3}\left(1 / \theta_{e}\right)+K_{1}\left(1 / \theta_{e}\right)}{4 K_{2}\left(1 / \theta_{e}\right)}-1\right]
$$

varies from $3 / 2$ for nonrelativistic electrons to 3 for fully relativistic electrons, and $K_{n}$ are modified Bessel functions of the $n$th order. The energy density of power-law electrons is

$$
u_{\mathrm{pl}} \approx N_{\mathrm{pl}} m_{e} c^{2} \frac{p-1}{p-2} \gamma_{\min }^{2-p}
$$


for $p>2$. So the number density of power-law electrons is determined by $u_{\mathrm{pl}}=\eta u_{\mathrm{th}}$, which gives

$$
N_{\mathrm{pl}}=\frac{p-2}{p-1} \gamma_{\min }^{p-2} \eta a\left(\theta_{e}\right) \theta_{e} N_{\mathrm{th}} .
$$

We obtain a second relation from the condition that the power-law distribution should match smoothly onto the thermal distribution:

$$
n_{\mathrm{th}}\left(\gamma_{\min }\right)=n_{\mathrm{pl}}\left(\gamma_{\min }\right) .
$$

This condition is natural since the nonthermal electrons are presumably accelerated out of the thermal pool. We solve equations (12) and (13) to obtain $N_{\mathrm{pl}}$ and $\gamma_{\min }$ simultaneously as a function of radius.

When we consider models for flares $(\S 4)$, we have $p<2$. In this case, the formula corresponding to eqs. (11)-(12) are

$$
u_{\mathrm{pl}} \approx N_{\mathrm{pl}} m_{e} c^{2} \frac{p-1}{2-p} \gamma_{\max }^{2-p},
$$

and

$$
N_{\mathrm{pl}}=\frac{2-p}{p-1} \gamma_{\max }^{p-2} \eta a\left(\theta_{e}\right) \theta_{e} N_{\mathrm{th}} .
$$

The value of $\gamma_{\max }$ depends on the details of electron acceleration which are not understood. We treat it as a free parameter, and adopt the minimum $\gamma_{\max }$ required to fit the X-ray spectrum of the flare detected by Chandra.

\subsection{Synchrotron and Inverse-Compton Emissions}

After determining the distribution of both thermal and power-law electrons, we calculate their radiation. Following Mahadevan, Narayan \& Yi (1996), the optically thin synchrotron emissivity of a relativistic Maxwellian distribution of electrons is

$$
j_{\nu, \mathrm{th}}=\frac{4 \pi N_{\mathrm{th}} e^{2}}{\sqrt{3} c K_{2}\left(1 / \theta_{e}\right)} \nu M\left(x_{M}\right),
$$

where

$$
x_{M} \equiv \frac{2 \nu}{3 \nu_{b} \theta_{e}^{2}}, \quad \nu_{b} \equiv \frac{e B}{2 \pi m_{e} c},
$$

with $M\left(x_{M}\right)$ given by

$$
M\left(x_{M}\right)=\frac{4.0505}{x_{M}^{1 / 6}}\left(1+\frac{0.40}{x_{M}^{1 / 4}}+\frac{0.5316}{x_{M}^{1 / 2}}\right) \exp \left(-1.8899 x_{M}^{1 / 3}\right) .
$$


The synchrotron absorption coefficient $\alpha_{\text {th }}$ is related to the emissivity via Kirchoff's law.

For the emissivity of power-law electrons we use the following expressions (Westfold 1959; Blumenthal \& Gould 1970), which are more exact than the analytically integrated

formula given in Özel et al. (2000), especially for frequencies near the "cutoff" and the "break" in the power-law distribution:

$$
j_{\mathrm{pl}}=\frac{1}{2}(p-1) N_{\mathrm{pl}}\left(\frac{\sqrt{3} e^{2}}{2 c}\right) \nu_{b}\left(\frac{2 \nu}{3 \nu_{b}}\right)^{(1-p) / 2} H(p)\left[G\left(x_{2}\right)-G\left(x_{1}\right)\right],
$$

where $x \equiv \nu / \nu_{c}, \nu_{c}=(3 / 2) \nu_{b} \gamma^{2}$. The quantities $x_{1}$ and $x_{2}$ correspond to the minimum and maximum Lorentz factors of the power-law electrons. In the above equation,

$$
\begin{aligned}
H(p) & =\int(\sin \alpha)^{(1+p) / 2} g(\alpha) d \Omega_{\alpha} \\
& =2 \pi \sqrt{\pi} \Gamma\left(\frac{p+5}{4}\right) / \Gamma\left(\frac{p+7}{4}\right), \\
G\left(x_{1}\right) & =\int_{x_{1}}^{\infty} x^{(p-1) / 2} \int_{x}^{\infty} K_{5 / 3}(y) d y d x .
\end{aligned}
$$

For the absorption coefficient of power-law electrons, we use (Özel et al. 2000)

$$
\alpha_{\nu, \mathrm{pl}}=C_{\mathrm{pl}}^{\alpha} \eta \frac{e^{2} N_{\mathrm{th}}}{c} a\left(\theta_{e}\right) \theta_{e}\left(\frac{\nu_{b}}{\nu}\right)^{(p+3) / 2} \nu^{-1}
$$

with

$$
C_{\mathrm{pl}}^{\alpha}=\frac{\sqrt{3 \pi} 3^{p / 2}}{8} \frac{p-1}{m_{e}} \frac{\Gamma[(3 p+2) / 12] \Gamma[(3 p+22) / 12] \Gamma[(6+p) / 4]}{\Gamma[(8+p) / 4]} .
$$

We also consider the Comptonization of seed synchrotron photons, both those produced by thermal electrons and those produced by power-law electrons. The scattering electrons can also be either thermal or power-law. We adopt the method of Coppi \& Blandford (1990) to calculate the Comptonization. This method uses a local approximation, but it

gives reasonable results when compared with the more careful Comptonization calculations of Narayan, Barret, \& McClintock (1997).

\subsection{Radiative Transfer Calculation}

To calculate the spectrum, we adopt the "plane parallel rays" method (Mihalas 1978; Özel et al. 2000). In this method, the equation of radiative transfer for a time-independent, spherically symmetric flow is written for plane parallel rays of varying impact parameters 
through the flow and solved along these rays (Mihalas 1978). We integrate the equation using the formal solution and the approximate boundary conditions for a nonilluminated atmosphere (Mihalas 1978). We carry out the integral along rays with impact parameters up to $\sim 5000 R_{s}$, beyond which the electron temperature is too low for significant synchrotron emission. The total flux is then obtained by integrating over all impact parameters. See Özel et al. (2000) for details.

\section{Quiescent State Spectra}

Figure 1 shows the spectral data on Sgr A* that we wish to explain. The main challenges for a model are (i) to explain the submm bump and the excess radio emission at low frequencies, (ii) to satisfy the stringent upper limit in the infrared, and (iii) to explain the low X-ray flux in quiescence (the lower "bowtie" in Fig. 1), the fact that most of the quiescent X-ray flux is spatially resolved, and that it has a relatively soft X-ray spectrum. We wish to explain all these facts while also satisfying the density measured by Chandra at the accretion radius, and the upper limit on the density of the gas near the black hole as deduced from the linear polarization data of Aitken et al. (2000) and Bower et al. (2003).

The solid line in Fig. 1 shows the spectrum corresponding to a RIAF model of Sgr A*, for which the flow parameters vary with radius as shown in Fig. 2. The parameters in the model are $\alpha=0.1$, plasma $\beta=10$, i.e., the magnetic energy density is $10 \%$ of the thermal energy density, $\dot{M}_{\text {out }} \approx 10^{-6} M_{\odot} \mathrm{yr}^{-1}, s=0.27, \delta=0.55, p=3.5$, and $\eta=1.5 \%$.

Fig. 1 shows that the model fits the spectrum of Sgr A* fairly well, including the low-frequency radio data, the submm-bump, and the Chandra X-ray emission. The low frequency radio emission is self-absorbed synchrotron emission from power-law electrons in the accretion flow. As discussed by Özel et al. (2000), this part of the spectrum is determined by a very unusual source function, namely $S=j_{\mathrm{pl}} / \alpha_{\mathrm{th}}$ (since at these frequencies $\alpha_{\mathrm{th}} \gg \alpha_{\mathrm{pl}}$ while $j_{\mathrm{pl}} \gg j_{\text {th }}$ ). Mahadevan (1998) and Özel et al. (2000) used a canonical ADAF model to show that a small fraction of power-law electrons can explain the low-frequency radio data in Sgr A*. We find that the same is true even when $\dot{M}$ near the black hole is much less than in their models. The IR emission in our model is due to optically-thin synchrotron emission (and some SSC) by the same power-law electrons that produce the radio emission. By contrast, the submm-bump is primarily produced by thermal electrons in the inner parts of the RIAF. Finally, the X-ray emission is thermal bremsstrahlung emission. This emission originates at large radii far from the $\mathrm{BH}$, and is consistent with Chandra observations which indicate that the source is extended, with a size of $1^{\prime \prime}$. Moreover, because the Bondi accretion radius, $R_{a c c}$, is also $\approx 1$ ", the observed emission comes from the "transition region" between 
the ambient medium and the accretion flow, where the gas is being gravitationally captured by the central BH. To account for this, we have used Quataert's (2002) calculation of the bremsstrahlung spectra produced by correctly matching the accretion flow onto the ambient medium at radii $\approx R_{a c c}$.

It is important to check whether the model in Fig. 1 can satisfy the Faraday rotation measure constraint. We find that $\mathrm{RM} \approx 10^{7} \mathrm{rad} \mathrm{m}^{-2}$ if we integrate through the equatorial plane of the accretion flow, while $\mathrm{RM} \lesssim 5 \times 10^{5} \mathrm{rad} \mathrm{m}^{-2}$ at the region where most of the emission at $230 \mathrm{GHz}$ comes from if we integrate along the rotation axis. This large difference arises because in the latter case we only see the hot relativistic inner regions of the accretion flow (the RM produced by gas on the scales resolved by Chandra is only $\mathrm{RM} \approx 3 \times 10^{3} \mathrm{rad} \mathrm{m}^{-2}$ assuming equipartition $\mathrm{mG}$ magnetic fields). It should also be noted that these estimates are probably upper limits because they assume that the magnetic field is fully coherent and points along the line of sight (while the magnetic field in the accretion flow is actually predominantly toroidal). Given the above uncertainties, we are reasonably consistent with Bower et al.'s (2003) limit of $\mathrm{RM} \lesssim 2 \times 10^{6} \mathrm{rad} \mathrm{m}^{-2}$.

As noted above, the submm emission in our models is due to thermal electrons. Since the linear polarization of optically thick thermal synchrotron emission from a uniform medium is suppressed by $\exp (-\tau)$, where $\tau$ is the synchrotron optical depth, we also need to check whether our model can roughly account for the magnitude of the observed linear polarization $(\approx 10 \%)$. In addition, an interesting possibility is that this strong $\tau$ dependence could explain the observed rapid variation of linear polarization with frequency (from $\approx 7 \%$ at $230 \mathrm{GHz}$ to $\lesssim 2 \%$ at $112 \mathrm{GHz}$ ). We have calculated the linear polarization produced by the thermal electrons in our models; we include optical depth effects and Faraday rotation using the formula in Pacholczyk (1970), but have not calculated the conversion of linear to circular polarization. We assume that our line of sight is well out of the equatorial plane of the accretion flow so that the path length through the accretion flow at any radius $R$ is $\approx H(R)$, the scale height. Note that the Faraday rotation measure is a function of radius (and is $\sim 10^{6} \mathrm{rad} \mathrm{m}^{-2}$ for the radii that dominate the submm emission considered here).

In Fig. 3a we show $\tau$ as a function of radius for three frequencies and in Fig. 3b we show by open circles the degree of linear polarization as a function of frequency when Faraday rotation is neglected. At $\approx 400 \mathrm{GHz}$, the emission from all radii is optically thin, and the emission is calculated to be $\approx 70 \%$ linearly polarized for a uniform B-field. At $\approx 230 \mathrm{GHz}$, the maximal optical depth is $\tau \approx 2$, which suggests a factor of 10 suppression in the linear polarization. In fact, however, emission from radii with $\tau \lesssim 1$ contributes a large fraction of the flux, so we find that the net polarization (integrated over all radii) should still be very high, $\approx 59 \%$. At $\approx 112 \mathrm{GHz}$, a similar estimate yields a maximal polarization of $\approx 45 \%$. 
We thus conclude that thermal electrons can readily account for the observed level of linear polarization from Sgr A*. This is consistent with the work of Melia, Liu, \& Coker (2000) who use a slightly different method to conclude that thermal electrons in their spherical accretion models can account for the obseved level of linear polarization. However, because of the contribution from $\tau \lesssim 1$ regions, optical depth effects are unlikely to explain the large change in polarization with frequency. This must be due to other effects. One possibility is Faraday depolarization by the accretion flow itself (Bower et al 1999a, 2003; Quataert \& Gruzinov 2000). The filled circles in Fig. 3b show the degree of polarization when Faraday rotation is included. The strong suppression of polarization at $112 \mathrm{GHz}$ compared to $230 \mathrm{GHz}$ (consistent with that observed) is because the Faraday rotation angle is $\propto \nu^{-2}$ for the same rotation measure, and because the emission at low frequencies comes from radii where the rotation measure is actually somewhat larger. Other reasons for the change in polarization could include a change in the magnetic field geometry, or a change in the emission component (which in our models only occurs at $\$ 50 \mathrm{GHz}$ ).

The model has several parameters, $\alpha, \beta, \dot{M}_{\text {out }}, s, \delta, p, \eta$, and it would be useful to understand how the different parameters are determined and how well they are constrained. The viscosity parameter $\alpha$ and the magnetic field parameter $\beta$ are known approximately from numerical MHD simulations (e.g., Balbus \& Hawley 1998). We have assigned reasonable values to these parameters, $\alpha=0.1, \beta=10$, and have not considered variations. Although the outer mass accretion rate $\dot{M}_{\text {out }}$ is technically an independent parameter, in practice it is degenerate with $\alpha$ since the density of gas in the accretion flow scales roughly as $\dot{M} / \alpha$. Our choice of $\dot{M}_{\text {out }} / \alpha=10^{-5} M_{\odot} \mathrm{yr}^{-1}$ corresponds to a gas density of $\approx 130 \mathrm{~cm}^{-3}$ at $10^{5} R_{S}$, in agreement with Chandra observations (see $\S 1$ ). Once $\alpha$ is fixed, there is little freedom in $\dot{M}_{\text {out }}$.

The parameters $p$ and $\eta$ describe the distribution of the power-law electrons. The value of $\eta$ is determined by requiring the predicted spectrum to agree with the radio data. We have found that a value $\sim 1.5 \%$ gives a good fit to the data, more or less independent of $p$, while values a factor of several larger or smaller deviate noticeably from the data. Figure 4 shows how the model shown in Fig. 1 changes if we vary $p$, the slope of the power-law electron distribution function, while keeping $\eta$ fixed at $1.5 \%$. To highlight the important changes, only the synchrotron emission from thermal and power-law electrons is shown. Different values of $p$ give almost the same fit to the low-frequency radio spectrum, as found by Özel et al. (2000). However, the predicted spectra in the IR and X-ray bands show large variations. From this comparison, we conclude that $p \gtrsim 3.5$ is favored because otherwise the optically thin power law tail becomes too prominent and violates the IR and/or X-ray limits.

The parameters $s$ and $\delta$ behave as a pair. For each value of $s$, we determine $\delta$ by requiring 
the model to reproduce the peak flux in the sub-mm bump. For our baseline model with $s=0.27$ and $\dot{M}_{\text {out }} \approx 10^{-6} M_{\odot} \mathrm{yr}^{-1}$, the accretion rate onto the $\mathrm{BH}$ is $\dot{M} \approx 4 \times 10^{-8} M_{\odot} \mathrm{yr}^{-1}$. As we showed above, this model is reasonably consistent with the RM constraint. One way to decrease RM even more is to decrease the accretion rate onto the $\mathrm{BH}$ by increasing the power-law index $s$, defined in equation (1). Fig. 5 shows the effect of varying $s$ (adjusting $\delta$ appropriately). The dot-dashed line is the same as the dot-dashed line in Fig. 1, i.e., it corresponds to $s=0.27, \delta=0.55$. The dashed line is for $s=0.1$ and $\delta=0.1$. This model fits the spectrum very well, but its rotation measure is too large: $\mathrm{RM} \sim 3 \times 10^{8} \mathrm{rad} \mathrm{m}^{-2}$, which implies that the polarization would be completely suppressed at frequencies of order a few hundred GHz. In fact, this model is similar to the canonical ADAF model (Narayan et al. 1998; Özel et al. 2000), which is inconsistent with the radio polarization data. Lastly, the dotted line is for $s=0.4$ and $\delta=1.0$. This model significantly overpredicts the flux in the submm bump over the frequency range $10^{10.5}-10^{11.5} \mathrm{~Hz}$, as has been shown earlier by Quataert \& Narayan (1999) and Yuan, Markoff, \& Falcke (2002). In addition, because the RM is very low, the polarization would be very high even at low frequencies, in conflict with the observations.

\section{X-ray Flares}

The X-ray flares are the most dramatic result from the Chandra observations of Sgr A*. In this section we examine models for these flares within the context of RIAFs. Markoff et al. (2001) showed that the flares are probably due to enhanced electron heating or acceleration, rather than a change in the accretion rate onto the $\mathrm{BH}$; otherwise there is too much variation at lower frequencies compared to the observations. A possible analog of the Chandra flares are solar flares, in which magnetic energy is converted into thermal energy, accelerated particles, and bulk kinetic energy, giving rise to a burst of radiation (e.g., Priest \& Forbes 2000). Although it is empirically established that particle heating and acceleration are quite efficient in solar flares, it remains unclear whether particle acceleration is dominated by direct acceleration in current sheets, stochastic acceleration by turbulence, or shock acceleration (e.g., Miller 1998).

Since global MHD simulations of RIAFs find highly time-dependent dissipation of magnetic energy (e.g., Hawley \& Balbus 2002; Machida \& Matsumoto 2003; Igumenshchev et al. 2003), it is natural to suppose that solar flare-like acceleration events happen in the accretion flow close to the BH. We therefore focus on this possibility, but discuss a few more speculative ideas as well. We assume that there is (occasionally) enhanced particle acceleration in the inner region of the RIAF, at $\lesssim 10 R_{s}$. In this scenario, the timescale of the 
flare will be set by the accretion timescale at $\sim 10 R_{s}$, or the Alfven crossing time of large scale magnetic loops in this region, both of which are of order an hour. Some fraction of the magnetic energy in the flare will be used to heat the thermal electrons, while some fraction will accelerate electrons into a power-law distribution of the form

$$
\begin{gathered}
N(\gamma) d \gamma=N_{0} \gamma^{-p} d \gamma, \quad \gamma_{\min } \leq \gamma \leq \gamma_{c}, \\
N(\gamma) d \gamma=N_{0} \gamma_{c} \gamma^{-p-1} d \gamma, \quad \gamma_{\mathrm{c}} \leq \gamma \leq \gamma_{\max }, p \geq 1
\end{gathered}
$$

\subsection{Synchrotron Emission by Accelerated Electrons}

In our quiescent models of Sgr $\mathrm{A}^{*}$, the magnetic field at $\lesssim 10 R_{s}$ is $B \sim 20 \mathrm{G}$. With this magnetic field strength, electrons with Lorentz factors $\gamma \sim 10^{5}$ emit synchrotron radiation in Chandra's band. If there is a sufficient number of such electrons, i.e., if $p$ is small enough, then these electrons will produce a hard X-ray flare. We note in this connection that some calculations of acceleration in current sheets give quite small values of $p$; e.g., Larrabee et al. (2003) find $p=1$ while the numerical simulations of Nodes et al. (2003) give $p=1.1-1.5$.

The synchrotron cooling time for electrons emitting in Chandra's band $\left(\nu \approx 10^{18} \mathrm{~Hz}\right)$ is

$t_{c o o l} \approx 20 B_{20}^{-3 / 2} \mathrm{~s}$, much less than the duration of the flare. Cooling is thus quite important (Fig. 2) and there is a cooling break in the emitted spectrum below the X-rays. Note that from $\S 2.2$, the hardest power-law that can be produced by synchrotron emission above the cooling break is one with a photon index of $\Gamma=1.5$.

The thick-dashed line in Fig. 6 shows an example of fitting the X-ray flare using synchrotron emission. Electrons in $\mathrm{a} \approx 10 R_{S}$ region close to the $\mathrm{BH}$ are assumed to be accelerated into a power-law with $\gamma_{\max } \approx 10^{6}, \eta=5.5 \%$, and $p=1$. The cooling break is evident at $\nu \approx 10^{13} \mathrm{~Hz}$. In Chandra's band the photon index is $\Gamma=1.5$, as explained above. This spectrum is consistent with the largest flare observed by Chandra, and is also consistent with the average spectrum of all the flares observed thus far with Chandra: $\Gamma=1.3_{-0.4}^{+0.5}$ (Baganoff 2003b).

It is important to note that the fraction of the energy in power-law electrons in the flare model, $\eta=5.5 \%$, is not that different from that in our quiescent model, $\eta=1.5 \%$ (Fig. 1). Nonetheless, the synchrotron contribution in the X-rays is much larger because the electron spectral index $p$ is assumed to be smaller ( $p=1$ vs. $p=3.5$ ). Finally, we note from Fig. 6 that during the flare, only the X-ray flux changes significantly; the radio, submm, 
and IR remain essentially constant. This is consistent with the current observations and is due to the fact that the total number of lower energy electrons $(\gamma \sim 1-10)$ that emit in the radio-IR is essentially unchanged during the flare. Of course, the flare may heat the thermal electrons at the same time that it produces the hard power-law distribution. In this case, there could be an increase in the submm bump flux coincident with the X-ray flare. In principle, coordinated observations could help determine the fraction of the flare energy going into thermal electrons versus power-law electrons.

It is interesting to consider whether the constraint highlighted above, namely $\Gamma>1.5$ because of the cooling break, can be circumvented. Within the synchrotron model, the only way is by having a hard electron energy distribution and not having the electrons cool. This means that the magnetic field should be sufficiently weak that the cooling time is $\gtrsim 1$ hour, the flare duration. This in turn requires $B \lesssim 1 \mathrm{G}$ in the emitting region.

We have considered various ways of reducing the magnetic strength in our models. The most natural way is to decrease the accretion rate. At fixed $\beta, B \propto \dot{M}^{1 / 2}$ so a factor of $\gtrsim 100$ decrease in $\dot{M}$ is required. As explained in $\S 3$, however, we have found it difficult to explain the quiescent spectrum of Sgr A* with such a low $\dot{M}$. Another possibility is that the "typical" field strength close to the $\mathrm{BH}$ is indeed $\sim 20 \mathrm{G}$ as estimated above, but that the emission we see is dominated by a region of much lower magnetic field strength.

One region where such a low magnetic field is possible is in current sheets in the accretion flow. In magnetic reconnection the strength of the magnetic field in the region where electrons are accelerated is much lower than in the ambient medium; in fact, $B \sim 0$ at the center of the current sheet (e.g., Priest \& Forbes 2000). If a reconnection event is "strong" enough to ensure that there is a large number of accelerated electrons, and if the newly accelerated electrons can be trapped in the low-B region for an accretion time, one could in principle obtain X-ray spectra with $\Gamma<1.5$ from synchrotron emission. Due to the uncertainties in reconnection theory, it is unclear whether the above two conditions can be met.

More plausibly, the emission could be dominated by the corona of the RIAF or an outflow/jet driven from the surface of the accretion flow. Since the magnetic field strength will decrease with distance from the midplane of the flow, the cooling break would be less important in these regions (though the emission still must occur sufficiently close to the $\mathrm{BH}$ to explain the duration of the flare). This model requires the acceleration of electrons to be less efficient in the bulk of the RIAF where the magnetic field strength is higher, otherwise that region would dominate the emission.

The thin dashed line in Fig. 6 shows a concrete example in which synchrotron emission from a region with $B \approx 0.3 \mathrm{G}$ produces an X-ray flare (in current sheets or outflow/jet). We 
assume that electrons have been accelerated with $p=1.2$ and $\eta=9 \%$. For these parameters, the ratio of the number of power-law electrons to the thermal electrons is only $\sim 10^{-6}$. Note that there is no cooling break in the spectrum; the break at high photon energies is because we took $\gamma_{\max } \sim 10^{7}$.

\subsection{Inverse Compton Emission by Accelerated Electrons}

Synchrotron emission is a viable explanation for the X-ray flares only if acceleration of very high $\gamma$ electrons is quite efficient. Alternatively, lower energy electrons can Comptonscatter synchrotron photons to produce an X-ray flare. Since the frequency of an up-scattered photon is $\sim \gamma^{2} \nu$, the required $\gamma \sim \sqrt{10^{18} / 10^{12}} \sim 10^{3}$. From Fig. 2, we see that cooling is not important for such electrons. Thus, in principle the spectrum can be much harder than that produced by the synchrotron models discussed in the previous section.

The dashed line in Fig. 7a shows an example of an IC model for the X-ray flares, in which we assume that electrons in a region $\approx 2.5 R_{S}$ in size are accelerated into a distribution with $p=0.5$. The model requires $\eta \approx 120 \%$ in order to produce the luminosity of the flare. This means that about $40-50 \%$ of the electrons in the volume are accelerated into the power-law distribution. The model reproduces the observed X-ray spectrum reasonably well. It also predicts a factor of a few variability in the submm and IR. This is because the accelerated electrons responsible for the X-ray flare also produce significant synchrotron emission at lower frequencies.

The value of $p$ in the above model is rather extreme since it corresponds to a very hard energy distribution, so one would like to check whether softer energy distributions are also acceptable. Fig. 7b compares the above model with another one with $p=1.1$, $\eta=100 \%$, and $\gamma_{\max }=630$. This model requires $90 \%$ of the electrons to be in the power-law distribution, which is rather extreme. The model is also very close to violating the IR limit on the spectrum.

\subsection{A Two-Phase Medium?}

The spectra of the observed X-ray flares are interestingly close to that produced by thermal bremsstrahlung emission, $\Gamma \approx 1$. The problem with invoking bremsstrahlung to explain the observed rdiation is that, to produce a luminosity of $L_{35} 10^{35} \mathrm{ergs} \mathrm{s}^{-1}$ from a sphere of radius $R$, the gas density must be $n \approx 10^{9} L_{35}^{1 / 2} T_{e, 10}^{1 / 4}\left(R / 10 R_{S}\right)^{-3 / 2} \mathrm{~cm}^{-3}$, where $T_{e, 10}=T_{e} /\left(10^{10} \mathrm{~K}\right)$. For comparison, the density in the inner $10 R_{S}$ for our quiescent model 
of Sgr $\mathrm{A}^{*}$ is $\approx 10^{6}-10^{7} \mathrm{~cm}^{-3}$ (Fig. 2). This makes it difficult to produce an X-ray flare using bremsstrahlung emission since it would require a large change in the gas density which would lead to a much larger increase in the emission at lower frequencies than is observed (see, however, Liu \& Melia 2002 who suggest that variations in $T_{e}$ and $n$ can be appropriately correlated so as to avoid this difficulty). Another difficulty with a bremsstrahlung interpretation is that bremsstrahlung emission is dominated by very large radii in the RIAF, $\sim R_{a c c}$, not small radii where the flare must occur (see $\S 3$ for our discussion of the quiescent emission).

One interesting way out of these difficulties is to consider the possibility that there is a two-phase medium in the accretion flow (see, e.g., Nayakshin \& Sunyaev 2003 for related ideas). If there is a cooler denser phase embedded in the hot RIAF, it could satisfy the above density requirement and give rise to bremsstrahlung emission. Pressure balance with the surrounding RIAF requires $n_{b} T_{b}=n_{i} T_{i}$ where $n_{i}, T_{i}$ are the number density and ion temperature in the RIAF, and $n_{b}, T_{b}$ are the density and temperature of the cooler "blob" (note that we assume thermal pressure balance; magnetic pressure could, however, be important; e.g., Kuncic, Celotti, \& Rees 1997)

We require $T_{b} \gtrsim 10^{8} \mathrm{~K}$ in order for the blob to produce hard X-ray emission observed by Chandra. It then follows that if the size of the blob is $R \sim 5 R_{S}$ it can produce bremsstrahlung with the required luminosity, $L \sim 10^{35} \mathrm{ergs} \mathrm{s}^{-1}$ (one can readily confirm that the blob is optically thin to free-free absorption and electron scattering). Note that neither bremsstrahlung nor synchrotron emission from the blob will produce any significant flux at frequencies lower than the X-ray, so the spectral fit at those frequencies will not be affected. The duration of the flare is presumably set by the time it takes the blob to fall into the black hole from $\sim 10 R_{S}$. The "blob" in this solution is notvery small and actually occupies a decent fraction of the volume of the accretion flow. In addition, almost all of the mass accretion occurs via the blob, not the hot RIAF. This means that the blob probably must be fed by some other source of gas in the external medium that is not observed by Chandra, e.g. the cold molecular material invoked by Nayakshin \& Sunyaev (2003; but note that our two-phase interpretation of the flare is completely different from theirs).

The primary problem with the bremsstrahlung idea is that it is not clear if a cool phase can be maintained at the required temperature of $\sim 10^{8} \mathrm{~K}$. Unlike in the ISM, there is no thermal instability in the background RIAF. In luminous AGN, a cool optically thick disk with $T \sim 10^{5} \mathrm{~K}$ is believed to coexist with a hot optically thin corona with $T \sim 10^{9} \mathrm{~K}$. Analogous optically thick material would have $T \sim 10^{3} \mathrm{~K}$ in $\operatorname{Sgr} \mathrm{A}^{*}$, much less than that required. Clearly, more work on the possible energetics and confinement of a "cool" $\sim 10^{8}$ $\mathrm{K}$ two-phase medium is required before this proposal can be considered a viable explanation 
for the Chandra observations.

\section{Summary and Discussion}

New observations of Sgr A* impose strong constraints on theoretical models for how gas accretes onto the black hole at the center of our Galaxy. In this paper, we have investigated how these observations can be understood in the context of radiatively inefficient accretion flows. The high level of linear polarization detected at $230 \mathrm{GHz}$ constrains the rotation measure through the accretion flow $\left(\lesssim 2 \times 10^{6} \mathrm{rad} \mathrm{m}^{-2}\right)$ and thus puts an upper limit on the density of gas near the BH. This argues for an accretion rate much less than the Bondi rate of $\sim 10^{-5} M_{\odot} \mathrm{yr}^{-1}$. A similar conclusion has been reached by theoretical studies of the dynamics of RIAFs; in particular, numerical simulations show that very little of the mass supplied to the accretion flow at large radii actually reaches the black hole. Our baseline model has a net accretion rate onto the $\mathrm{BH}$ of $\dot{M} \approx 4 \times 10^{-8} M_{\odot} \mathrm{yr}^{-1}$. Phrased in terms of $\dot{M} \propto r^{s}$ or $\rho \propto r^{-3 / 2+s}$ this corresponds to $s \approx 0.27$ when we normalize the density and thus the accretion rate to that inferred from Chandra observations $\approx 1^{\prime \prime}$ from the $\mathrm{BH}$.

We have focused on the possibility that there are both thermal and nonthermal electrons in the accretion flow; this is natural since the accreting gas in RIAFs is a hot collisionless magnetized plasma. We calculate the emission from both types of electrons. Mahadevan (1998) and Özel et al. (1999) showed that the low-frequency radio spectrum, which was under-predicted in the original ADAF models (Narayan et al. 1998), can be explained if a small fraction $\eta$ of the electron energy resides in the power-law tail. We confirm that result for the RIAF models considered here, and find that a fraction $\eta \sim 1.5 \%$ is sufficient to explain the data (Fig. 1). If the maximum Lorentz factor of the electrons is reasonably large, we find that the power-law index of the electron distribution must satisfy $p \gtrsim 3.5$; otherwise synchrotron emission from the power-law electrons violates IR and X-ray (quiescent state) observations (Fig. 4). The thermal electrons in our model are responsible for the submm emission and can account for the observed level of linear polarization ( $§ 3 \&$ Fig. 3). This is consistent with the work of Melia, Liu \& Coker (2000).

It is interesting to note that there may be independent observational evidence for nonthermal electrons in RIAFs. McConnell et al. (2000) combined data from the COMPTEL experiment on $C G R O$ with data from both BATSE and OSSE to produce a broadband $\gamma$-ray spectrum for the hard state of Cyg X-1 (from $50 \mathrm{keV}$ up to $\sim 5 \mathrm{MeV}$ ). The data clearly show a hard tail at high energies; this can be explained as synchrotron from electrons with a steady state spectral index of $p=4.5$. Since cooling of the electrons is undoubtedly important at such high energies, the spectral index of the injected electrons would be $p=3.5$, 
similar to that found here. The hard state of Cyg X-1 can be naturally explained in terms of an ADAF-like model (Esin etal. 1998). Therefore, even though the luminosities are very different, the basic physics of the gas flow and particle acceleration may be similar in Cyg $\mathrm{X}-1$ and $\operatorname{Sgr} \mathrm{A}^{*}$.

Nonthermal electrons may also account for the X-ray flares observed by both Chandra and XMM-Newton from Sgr A*, as proposed by Markoff et al. (2001). Specifically, if some of the electrons close to the $\mathrm{BH}\left(\lesssim 10 R_{S}\right)$ are occasionally accelerated into a power-law tail with a very hard power-law index ( $p \sim 1$, rather than $p \sim 3.5$ as is required to explain the radio emission), synchrotron emission or synchrotron self-Compton emission by these accelerated electrons can produce X-ray flares similar to those observed ( $\$ 4$; Figs. 6-8). In synchrotron models for the X-ray flare, the required energy in power-law electrons is $\sim 10 \%$ of the available electron thermal energy in the inner $\sim 10 R_{S}$. By contrast, in IC models it is closer to $100 \%$, i.e., nearly all of the available energy (and electrons) must go into a powerlaw tail. This is a somewhat stringent requirement and so we favor the synchrotron models. In addition, the upper limits on the IR emission from Sgr A* are much more difficult to satisfy in IC models because such a large fraction of the electrons are accelerated (compare Figs. $6 \& 7$ ); the IR limits require that the maximum Lorentz factor in IC flares must be small, $\gamma_{\max } \lesssim 10^{3}$, for which there is no natural explanation.

Our synchrotron flare models in Fig. 6 produce no change in the radio-IR flux during the flare; this need not, however, be a robust feature of "synchrotron" flares. If some of the ambient thermal electrons are heated during the flare (which we have not accounted for in Fig. 6), they will produce additional submm-IR flux, leading to correlated radio-X-ray variability. This could produce variability similar to that expected in SSC models, making it difficult to pin down the emission mechanism using variability (though the continued absence of any lower frequency counterparts to the X-ray flares would, we believe, favor synchrotron models).

In addition to the solar-like flare models, we have also briefly considered a very different, more speculative, explanation for the observed X-ray flares, namely the flares could be due to bremsstrahlung emission from cooler, denser gas embedded in the hot RIAF ( $\$ 4.3)$. Such a model would nicely explain the very hard X-ray spectra observed (photon index $\Gamma \sim 1$ ). Although in principle viable, this possibility hinges on an unusual two-phase medium: the "cool" gas must still be quite hot, with $T \sim 10^{8} \mathrm{~K}$, to produce hard X-ray emission (the background RIAF has $T_{p} \sim 10^{11}-10^{12} \mathrm{~K}$ close to the $\left.\mathrm{BH}\right)$. More work is needed to determine whether this is physical.

One feature of our calculations should be pointed out: the model shown in Fig. 1 has $\dot{M} \approx 4 \times 10^{-8} M_{\odot} \mathrm{yr}^{-1}$ close to the black hole. If the outer radius of the RIAF is at 
$\approx R_{a c c} \approx 10^{5} R_{S}$, and if we set the density at this radius to the value measured by Chandra, then it implies $s \approx 0.27$, where $\dot{M} \propto r^{s}$. This value of $s$ is smaller than the values $\approx 0.5-1$ found in numerical simulations of RIAFs (e.g., Hawley \& Balbus 2002; Igumenshchev et al. 2003). We have found it difficult to account for the submm emission of Sgr A* with a larger $s$ and thus a lower $\dot{M}$ (Fig. 5). The reason is that for much smaller $\dot{M}$, the electron temperature has to be larger to produce a luminosity comparable to that observed; the hot electrons then occupy such a large volume that they significantly overpredict the $10^{10.5}-10^{12}$ Hz emission (Fig. 5).

There are several possible resolutions of this "problem": (1) the outer radius of the rapidly rotating part of the RIAF may be $\ll R_{a c c}$ (because of low angular momentum at large radii). For a given gas density close to the $\mathrm{BH}$ (from the $\mathrm{RM}$ constraint) and a given gas density at $\approx 1^{\prime \prime}$ (from Chandra observations), a larger value of $s$ would then be required, more consistent with the simulation results. (2) The electron thermodynamics could be more complicated then we consider. For example, the electron heating $(\delta)$ could be stronger in the inner part of the accretion flow close to the $\mathrm{BH}\left(\lesssim 10 R_{S}\right)$ than it is at larger radii. In this case, much lower $\dot{M}$ is consistent with the data because the volume occupied by the hottest electrons is relatively small and so the submm emission is less prominent.

For simplicity, we have taken all of the parameters such as $\alpha, \beta, \delta, \eta, p$ to be independent of radius. There is then not much freedom in the choice of parameter values in our model, with two exceptions: 1) The value of $p$ (the spectral index of injected power-law electrons) in the quiescent model is not strongly constrained; it just has to be $>3.5$. 2) Similarly, the maximum Lorentz factor $\gamma_{\max }$ of the power-law electrons in the synchrotron model of flares only has a lower limit $\left(\sim 10^{6}\right)$, but it could be quite a bit larger; in the latter case, the predicted flare spectrum would extend to higher frequencies than shown in Fig. 6.

Finally, we note that instead of the accretion flow alone producing all the radiation in Sgr A*, a jet may be responsible for some of the observed emission (e.g., Falcke et al. 1993; Falcke \& Markoff 2000). In particular, Yuan, Markoff, \& Falcke (2001; 2003) have proposed a coupled jet-RIAF model. In their model, the underlying accretion flow is described by a RIAF. Close to the $\mathrm{BH}$, a fraction of the accreting material is ejected to form a jet (outflows are seen in MHD simulations of RIAFs, though they are not yet particularly well collimated). The physical quantities in the jet are self-consistently matched onto the underlying RIAF (see Yuan et al. 2001), and the spectral fit to Sgr A* is satisfactory. As in other AGN, the self-absorbed synchrotron emission from the outer part of the jet fits the low-frequency radio spectrum of Sgr A* (that we have ascribed to non-thermal electrons in the RIAF in our model); the jet can also account for the high level of circular to linear polarization observed at low frequencies (Beckert \& Falcke 2002). 
In jet-RIAF models, the synchrotron emission from the base of the jet close to the $\mathrm{BH}$ dominates over the underlying RIAF and accounts for the submm-bump (because of effective electron heating in the shock front at the base of the jet). In addition, Markoff et al. (2001) proposed that the X-ray flares observed by Chandra could be understood as inverse Compton or synchrotron emission from electrons heated or accelerated in this region. Our synchrotron and SSC models are quite similar to these ideas, although the geometry is different.

While the jet-RIAF model is successful in explaining observations of Sgr $\mathrm{A}^{*}$, we have shown in the present paper that a pure RIAF model is also equally successful. An important question is to determine which dynamical component is responsible for which part of the observed emission. One possibility is to assess whether the coherent magnetic field needed to produce $\approx 10 \%$ linear polarization in the submm is consistent with that expected in the accretion flow, or whether it requires an additional component that could be attributed to the acceleration region of a jet. Another promising possibility is simultaneous multiwavelength observations in the radio-submm and X-ray. In a jet one would expect submm variability to lead the radio (if, e.g., variability is produced by shocks propagating down the jet), while if the accretion flow dominates the emission, one would expect little correlation (if variability

is due to local turbulence in the flow) or that the radio would lead the submm (if variability is due to changes in the accretion rate).

F.Y. and R.N. were supported in part by NASA grant NAG5-10780, NSF grant AST 9820686 and AST 0307433. E.Q. was supported in part by NASA grant NAG5-12043, NSF grant AST-0206006, and an Alfred P. Sloan Foundation Fellowship. We thank Fred Baganoff, Jun Lin, Sera Markoff, and the anonymous referee for useful comments.

\section{REFERENCES}

Agol, E. 2000, ApJ, 538, L121

Aitken, D.K., Greaves, J.S., Chyrsostomou, A. et al., 2000, ApJ, 534, L173

Baganoff, F. K. 2003a, to be published in Astron. Nachr., Vol. 324, No. S1 (2003), Special Supplement "The central 300 parsecs of the Milky Way", Eds. A. Cotera, H. Falcke, T. R. Geballe, S. Markoff

Baganoff, F. K. 2003b, invited talk in AAS HEAD meeting \#35

Baganoff, F. K., Maeda, Y., Morris, M. et al., 2003, ApJ, in press (astro-ph/0102151) 
Baganoff, F. K., Bautz, M. W., Brandt, W. N. et al., 2001, Nature, 413, 45

Balbus, S. A. \& Hawley, J. F. 1998, Reviews of Modern Physics, 70, 1

Beckert, T., \& Falcke, H. 2002, A\&A, 388, 1106

Blandford, R.D., \& Begelman, M.C. 1999, MNRAS, 303, L1

Blumenthal, George R.; Gould, R. J., 1970, Rev. Mod. Phys. 1970, 42, 237

Bondi, H. 1952, MNRAS, 112, 195

Bower, G. C., Backer, D. C., Zhao, J. H., Goss, M., \& Falcke, H. 1999a, ApJ, 521, 582

Bower, G. C., Falcke, H., \& Backer, D. C. 1999, ApJ, 523, L29

Bower, G. C., Falcke, H., Sault, R. J., \& Backer, D. C. 2002, ApJ, 571, 843

Bower, G. C., Wright, M. C. H., Backer, D. C., \& Falcke, H. 1999b, ApJ, 527, 851

Bower, G. C., Wright, M. C. H., Falcke, H., \& Backer, D. C. 2001, ApJ, 555, L103

Bower, G.C. et al. 2003, ApJ, 588, 331

Chandrasekhar, S. 1939, Introduction to the Study of Stellar Structure (Chicago: Univ. Chicago Press)

Coppi, P.S., \& Blandford, R.D. 1990, MNRAS, 245, 453

Esin, A. A., Narayan, R., Cui, W., Grove, J.E., \& Zhang, S., 1998, ApJ, 505, 854

Falcke, H., Goss, W. M., Matsuo, H., Teuben, P., Zhao, J. H., \& Zylka, R., 1998, ApJ, 499, 731

Falcke, H., Mannheim, K., and Biermann, P. L., 1993, A\&A, 278, L1

Falcke, H. \& Markoff, S., 2000, A\&A, 362, 113

Ghez, A.M. et al. 2003, to be published in Astron. Nachr., Vol. 324, No. S1 (2003), Special Supplement "The central 300 parsecs of the Milky Way", Eds. A. Cotera, H. Falcke, T. R. Geballe, S. Markoff (astro-ph/0303151)

Goldwurm A., et al. 2003, ApJ, 584, 751

Hawley, J. F. \& Balbus, S. A. 2002, ApJ, 573, 738 
Hornstein, S.D. et al. 2002, ApJ, 577, L9

Igumenshchev, I. \& Abramowicz, M. 1999, MNRAS, 303

Igumenshchev, I., Abramowicz, M., \& Narayan, R. 2000, 537, L27

Igumenshchev, Narayan, R., \& Abramowicz, M. A., 2003, ApJ, in press (astro-ph/0301402)

Koratkar, A. \& Blaes, O. 1999, PASP, 111, 1

Kuncic, Z., Celotti, A., \& Rees, M.J. 1997, MNRAS, 284, 717

Larrabee, D.A., Lovelace, R.V.E., \& Romanova, M.M. 2003, ApJ, 586, 72

Liu, S. \& Melia, F. 2001, ApJ, 561, L77

Liu, S. \& Melia, F. 2002, ApJ, 566, L77

Machida, M. \& Matsumoto, R. 2003, ApJ, 585, 429

Mahadevan, R. 1998, Nature, 394, 651

Mahadevan, R., Narayan, R., \& Yi, I. 1996, ApJ, 465, 327

Manmoto, T., Mineshige, S., Kusunose, M. 1997, ApJ, 489, 791

Markoff, S., Falcke, H., Yuan, F., \& Biermann, P. 2001, A\&A, 379, L13

McConnell, M.L. et al. 2000, ApJ, 543, 928

Melia, F. 1992, ApJ, 387, L25

Melia, F., \& Falcke, H. 2001, ARA\&A, 39, 309

Melia,F., Liu, S., \& Coker, R. 2000, ApJ, 545, L117

Melia,F., Liu, S., \& Coker, R. 2001, ApJ, 553, 146

Mihalas, D. 1978, Stellar Atmospheres (San Francisco: Freeman)

Miller, J.A. 1998, Spac. Sci. Rev. 86, 79

Narayan, R. 2002, in "Lighthouses of the Universe" eds. M. Gilfanov, R. Sunyaev et al., Springer-Verlag; p. 405

Narayan, R., Barret, D., \& McClintock, J. E. 1997, ApJ, 482, 448 
Narayan, R., Mahadevan, R., Grindlay, J.E., Popham, R. \& Gammie, C., 1998, ApJ, 492, 554

Narayan, R. \& Yi, I. 1994, ApJ, 428, L13

Narayan, R. \& Yi, I. 1995, ApJ, 444, 231

Narayan, R., Yi, I., \& Mahadevan, R., 1995, Nature, 374, 623

Nayakshin, S. \& Sunyaev, R. 2003, submitted to MNRAS (astro-ph/0302084)

Nodes, C., Birk, G.T., \& Schopper, R. 2003, Physics of Plasmas, in press (astro-ph/0301358)

Özel, F., Psaltis, D., \& Narayan, R. 2000, ApJ, 541, 234

Pacholczyk, A. G., 1977, Radio Astrophysics (San Francisco: W. H. Freeman \& Co.)

erence Paczyński, B., \& Wiita, P. J. 1980, A\&A, 88, 23

Priest, E. \& Forbes T. 2000, Magnetic Reconnection: MHD Theory and Applications (Cambridge: Cambridge Univ. Press)

Quataert, E. 2002, ApJ, 575, 855

Quataert, E. \& Gruzinov 1999, ApJ, 520, 248

Quataert, E. \& Gruzinov 2000, ApJ, 545, 842

erence Quataert, E., \& Narayan, R., 1999, ApJ, 520, 298

Ruszkowski, M. \& Begelman, M. 2002, ApJ, 581, 223

Schödel, R. et al. 2002, Nature, 419, 694

Serabyn, E., Carlstrom, J., Lay, O. et al., 1997, ApJ, 490, L77

Shakura, N. I.; Sunyaev, R. A, 1973, A\&A, 24, 337

Stone, J.M. \& Pringle, J.E. 2001, MNRAS, 322, 461

Stone, J., Pringle, J., \& Begelman, M. 1999, MNRAS, 310, 1002

Wardziński G., Zdziarski, A. A., 2001, MNRAS, 325,963; Erratum: 2001, MNRAS, 327, 351

Westfold, K.C. 1959, ApJ, 130, 241

Yuan, F. 1999, ApJ, 521, L55 
Yuan, F., Peng, Q., Lu, J., \& Wang, J. 2000, ApJ, 537, 236

Yuan, F., Markoff, S., \& Falcke, H., 2002, A\&A, 383, 854

Yuan, F., Markoff, S., \& Falcke, H., 2003, to be published in Astron. Nachr., Vol. 324, No. S1 (2003), Special Supplement "The central 300 parsecs of the Milky Way", Eds. A. Cotera, H. Falcke, T. R. Geballe, S. Markoff

Zhao, J.-H., et al. 2003, ApJ, 586, L29

Zylka, R., Mezger, P.G., \& Lesch, H. 1992, A\&A, 261, 119 


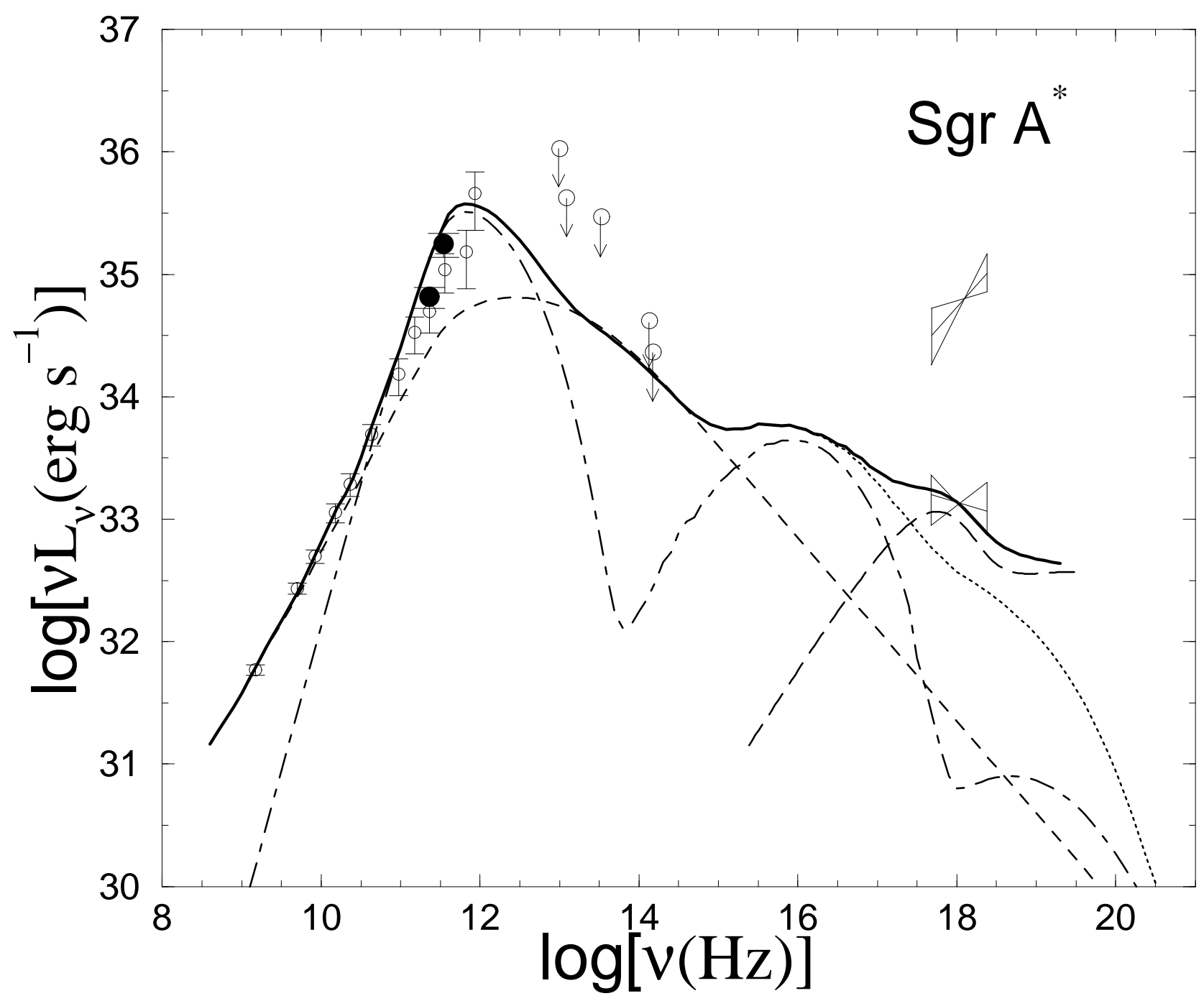

Fig. 1.- A model for the quiescent emission from Sgr A* with $s=0.27, \delta=0.55$. The accretion rate close to the $\mathrm{BH}$ is $\dot{M} \approx 4 \times 10^{-8} M_{\odot} \mathrm{yr}^{-1}$. The dot-dashed line is the synchrotron and IC emission by thermal electrons. The dashed line is the synchrotron emission by non-thermal electrons; the non-thermal electrons have $\approx 1.5 \%$ of the thermal energy with $p=3.5$. The dotted line is the total synchrotron and IC emission while the solid line includes the bremsstrahlung emission from the outer parts of the RIAF (shown by the long-dashed line); the latter component explains the extended quiescent X-ray source. The radio data are from Falcke et al. 1998 (open circles) and Zhao et al. 2003 (SMA; filled circles), the IR data are from Serabyn et al. (1997) and Hornstein et al. (2002). The two "bowties" in the X-ray are the quiescent (lower) and flaring (higher) data from Baganoff et al. (2001; 2003). 

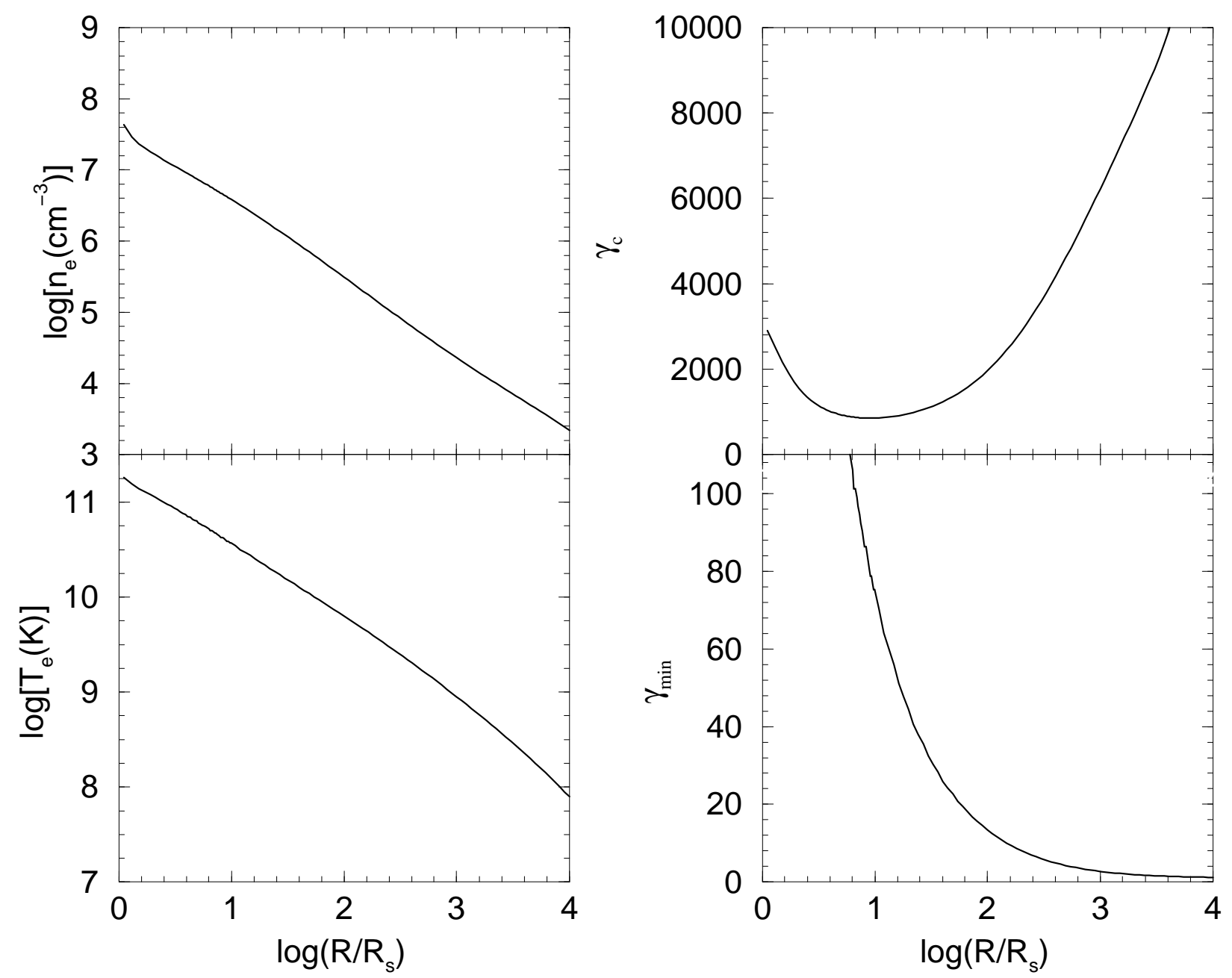

Fig. 2. - The radial profiles of electron density $n_{e}$, electron temperature $T_{e}$, cooling break Lorentz factor $\gamma_{c}$, and minimum Lorentz factor $\gamma_{\min }$ for the model shown in Fig. 1. 

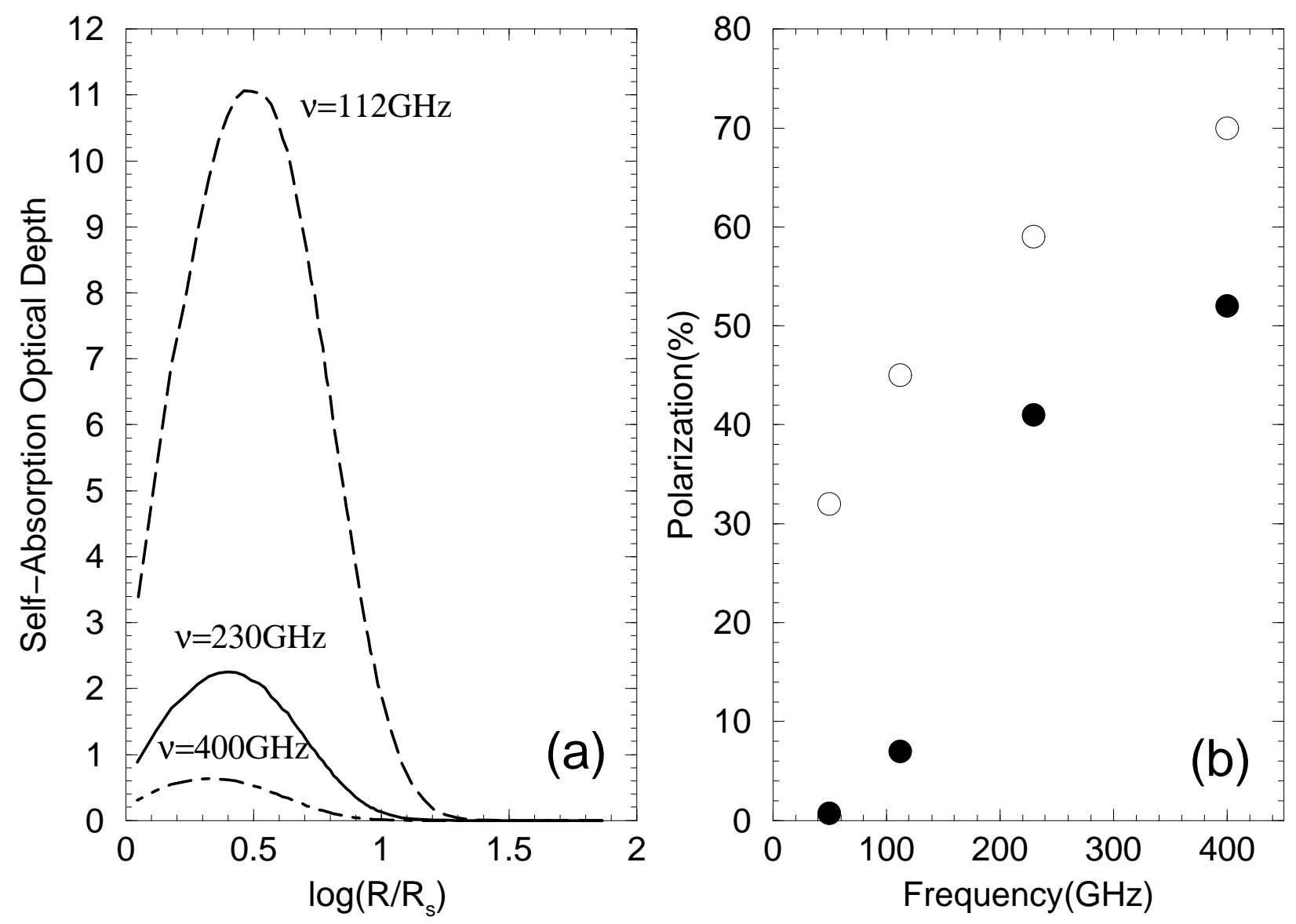

Fig. 3.- (a): Synchrotron self-absorption optical depth of thermal electrons as a function of radius at three frequencies for the model shown in Fig. 1. (b): The degree of linear polarization from thermal electrons at four frequencies with (filled circles) and without (open circles) Faraday rotation included. These values are upper limits because they assume a uniform B-field. 


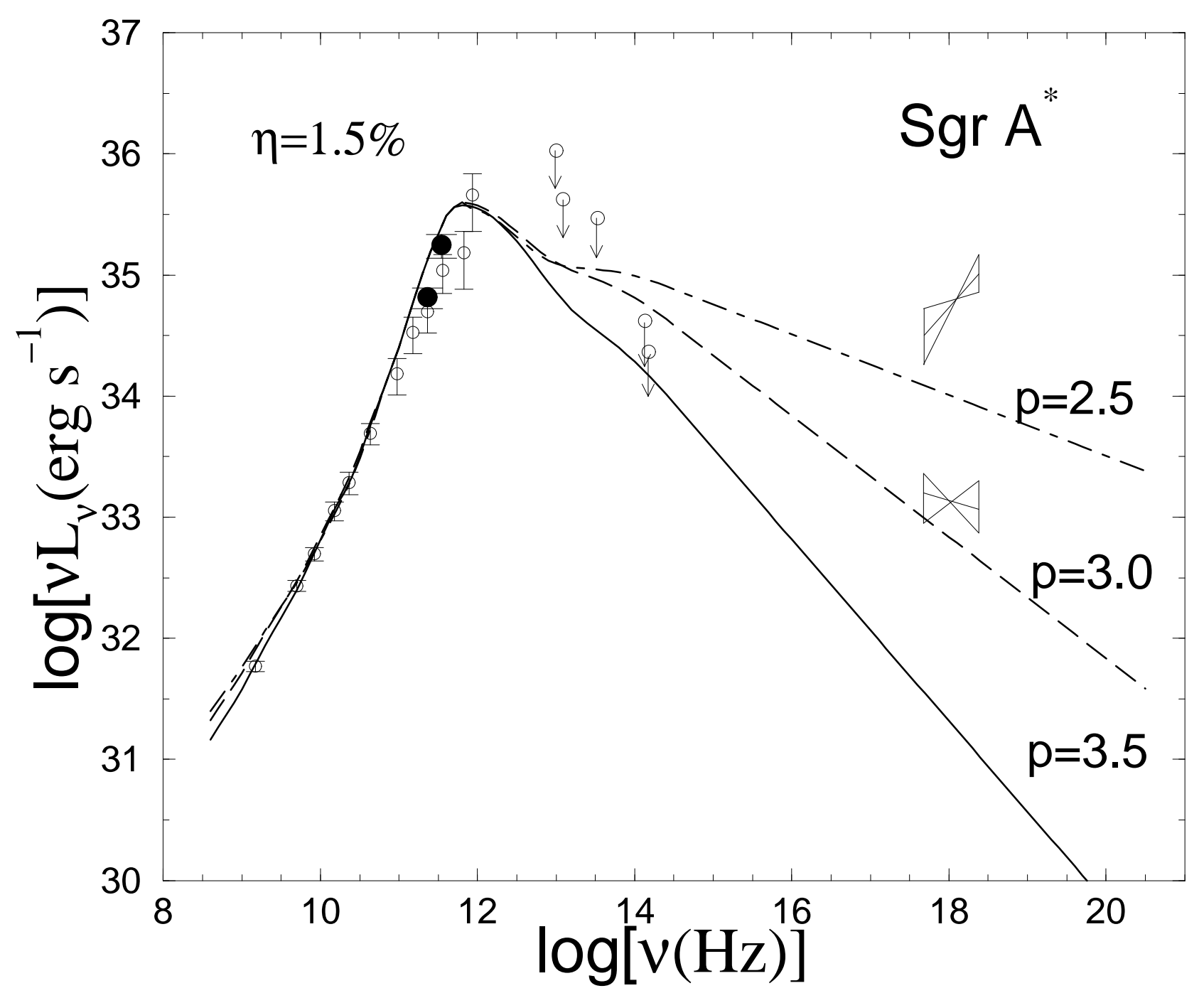

Fig. 4. - The effect of different electron power-law indices $p$ on the model shown in Fig. 1. Only the synchrotron emission from power-law and thermal electrons is shown. 


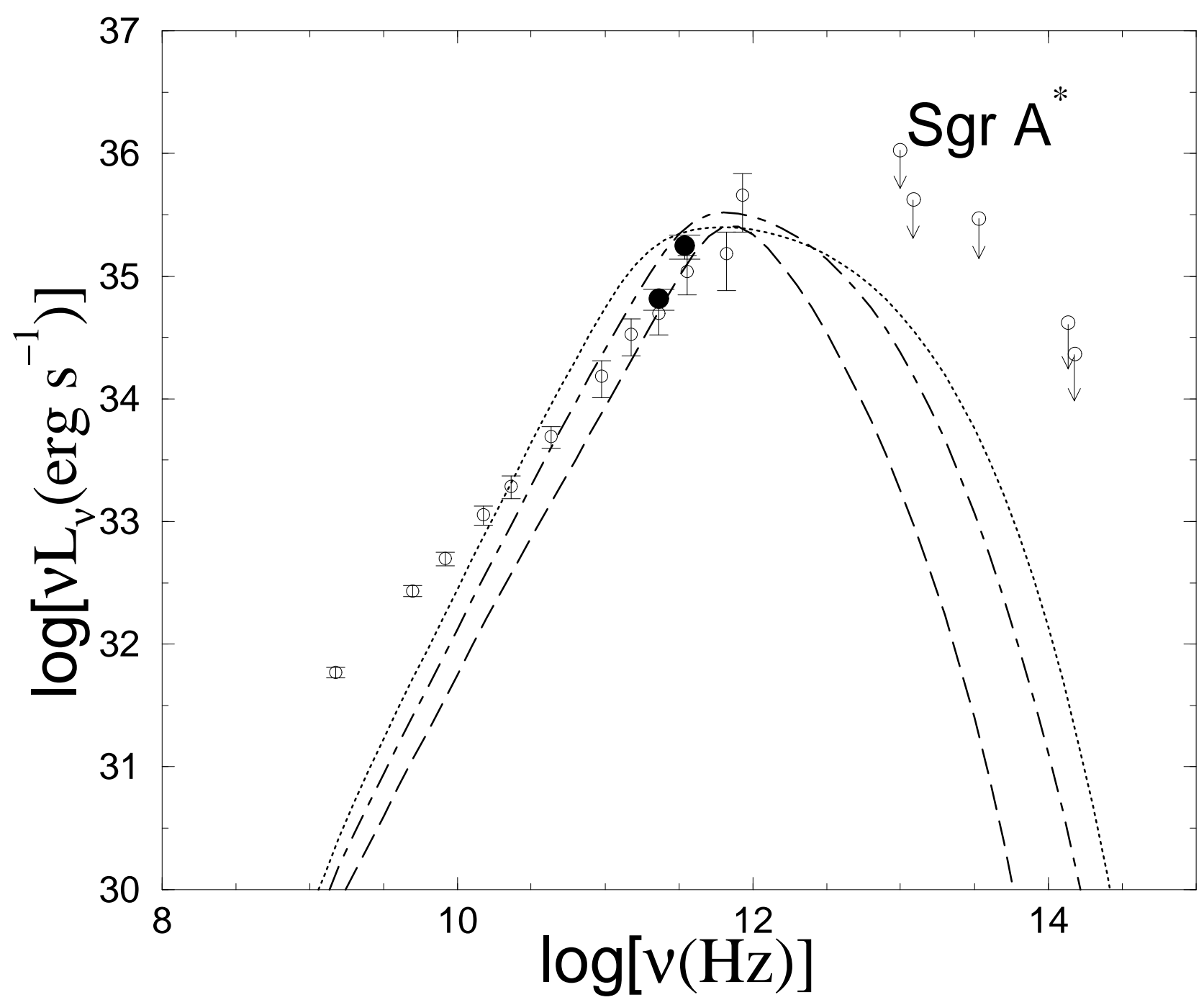

Fig. 5.- The effect of different values of $s$ and $\delta$ on the submm emission; since the thermal electrons dominate this emission, only their synchrotron emission is shown. The models correspond to $s=0.1, \delta=0.1$ (dashed line), $s=0.27, \delta=0.55$ (dot-dashed), $s=0.4, \delta=1$ (dotted). 


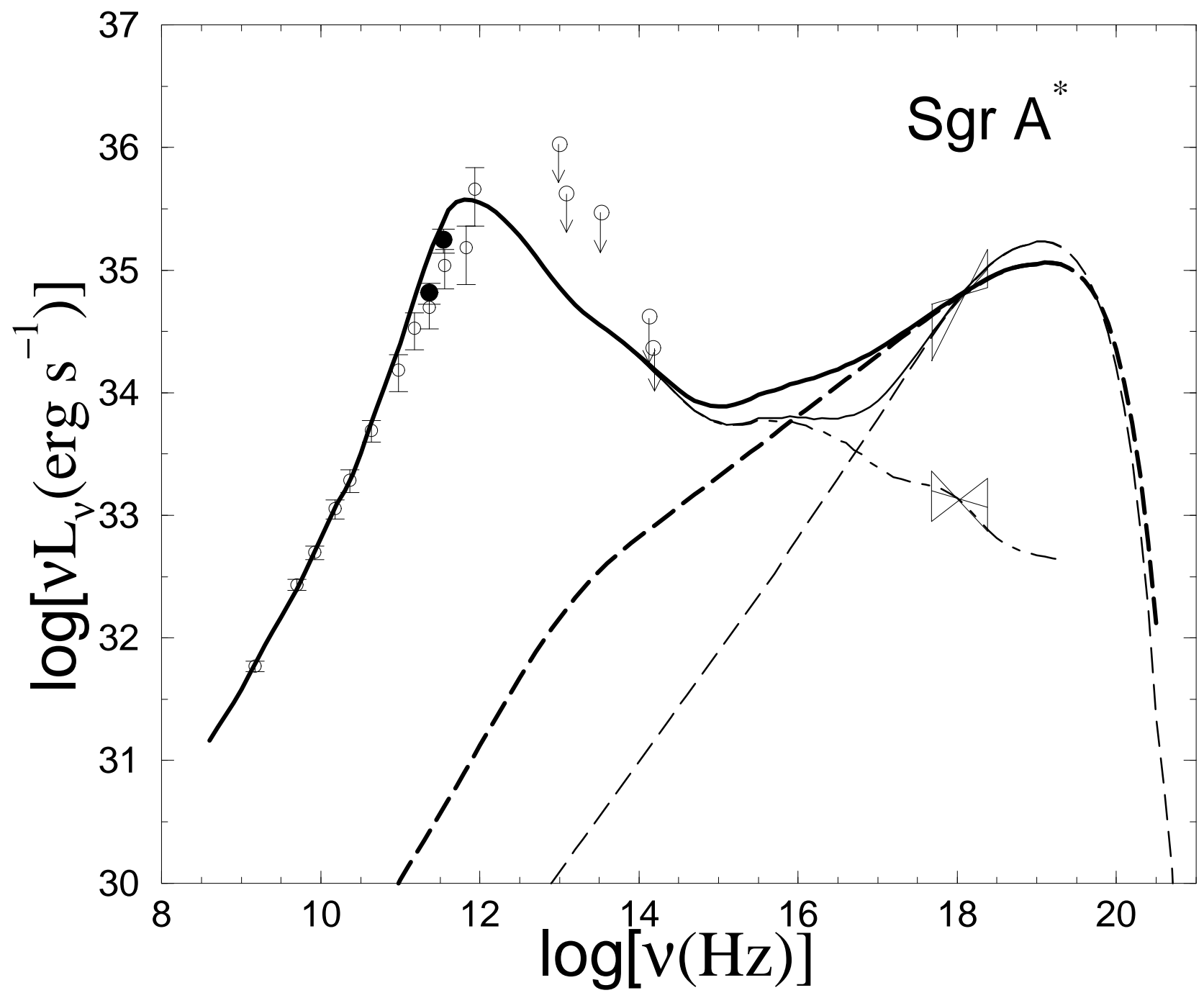

Fig. 6.- Synchrotron models for the X-ray flares from Sgr A*. The dot-dashed line is the quiescent emission from Fig. 1. The thick dashed line is the synchrotron flare due to power-law electrons with $\gamma_{\max } \approx 10^{6}, p=1$, and $\eta=5.5 \%$ (i.e., power-law electrons have $5.5 \%$ of the electron energy); the magnetic field in the RIAF close to the $\mathrm{BH}$ is $\sim 20 \mathrm{G}$ so there is a cooling break at $\sim 10^{13} \mathrm{~Hz}$. The thick solid line is the total emission during the flare (quiescent emission plus synchrotron flare). Finally, the thin lines (dashed and solid) correspond to a second synchrotron flare model in which $p=1.2$ and the magnetic field is only $0.5 \mathrm{G}$ so that there is no cooling break. 


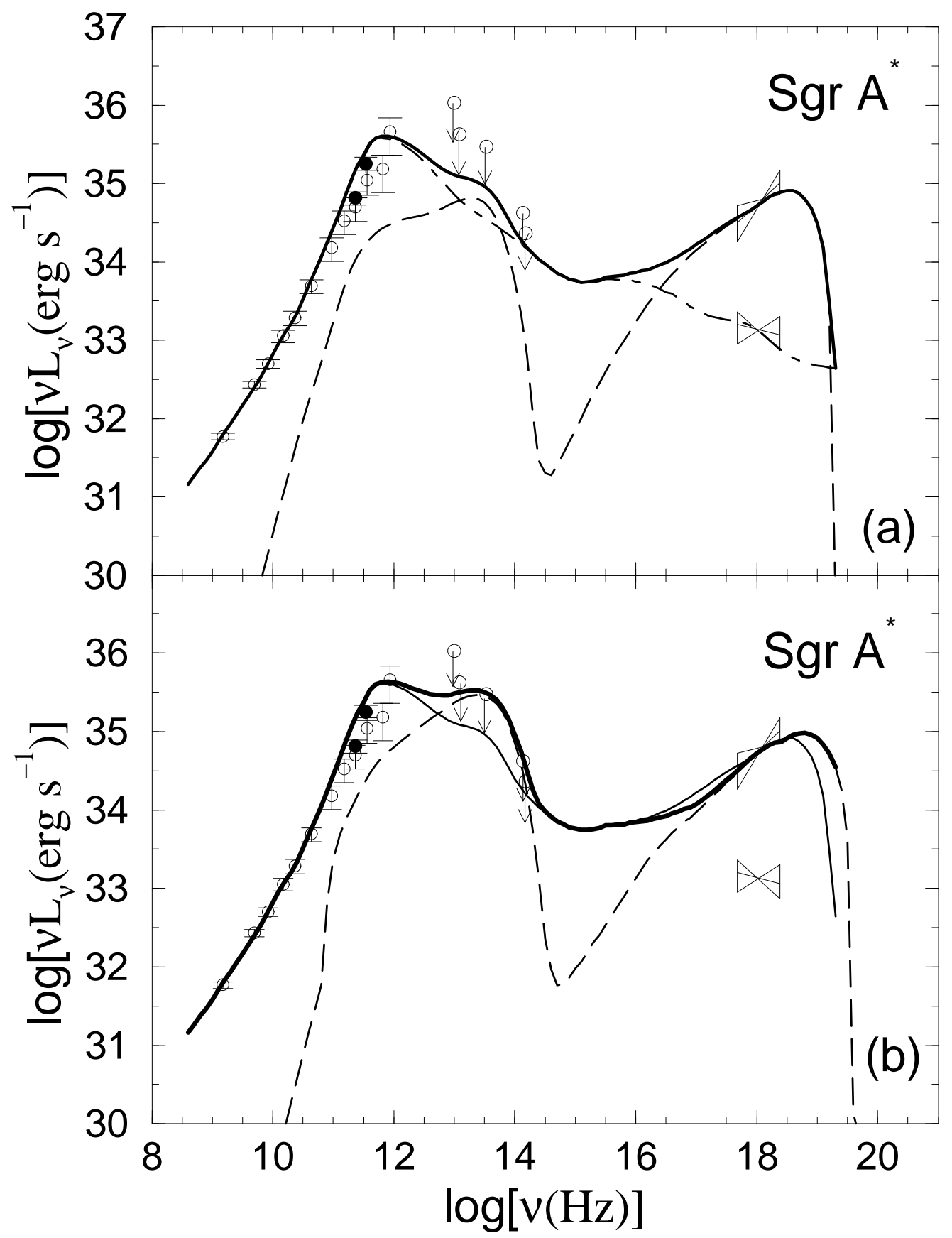

Fig. 7.- Two inverse Compton models for the X-ray flare. (a): The dot-dashed line is the quiescent emission from Fig. 1. The dashed line is the synchrotron and SSC emission in the "flaring region" - power-law electrons in a $\approx 2.5 R_{S}$ volume are accelerated with $p=0.5$ and $\eta=120 \%$. The solid line is the total emission during the flare. (b): The thin solid line is the IC flare model from (a). The dashed and thick solid lines are a second IC flare model with similar parameters except that $p=1.1$. 